\title{
An Incomplete Form of Shone's Complex With Double Orifice Mitral Valve
}

\author{
Petar Risteski, MD, Oana Bartos, MD, Aleksandra Miskovic, MD, Thomas Walther, MD, PhD \\ Department of Thoracic and Cardiovascular Surgery, University Hospital Frankfurt, Frankfurt am Main, Germany
}

\section{ABSTRACT}

We present the case of an adult male patient with an incomplete form of Shone's complex associated with bicuspid aortic valve and a double orifice mitral valve. Intraoperative inspection of the mitral valve showed double orifice configuration with a small, rudimentary left-sided mitral valve and a large, dominant, right-sided parachute mitral valve with Barlow-type of degeneration. The patient underwent reconstruction of both valves through a minimally invasive incision. At one year echocardiographic control both valves function normally.

\section{INTRODUCTION}

Shone's complex is a very rare congenital malformation defined by four left-sided heart defects, including coarctation of the aorta, subaortic obstruction, parachute mitral valve, and supravalvular mitral ring [Shone 1963]. However, a wider spectrum of patients has been enlisted as "incomplete Shone's complex," and these prevail in clinical practice [Shih 2019]. The rarity of such congenital malformation has been associated with under and later diagnosis in adulthood [Shehatha 2018]. Double orifice mitral valve (DOMV) is a very rare congenital defect defined as a single fibrous annulus with two orifices [Greenfield 1876].

\section{CASE REPORT}

A 50-year-old male presented in our clinic with progressive dyspnea. He had undergone surgical correction of post-ductal type of aortic coarctation at age 12, as part of an incomplete form of Shone's complex, including a parachute mitral valve and bicuspid aortic valve. Recent transthoracic echocardiography exam revealed moderate regurgitation of a bicuspid aortic valve and a severe regurgitation of the Barlow-type of mitral valve. A subtle analysis of the mitral valve in transesophageal echocardiography showed the presence of prolapse of multiple segments, including billowing

Received fuly 31, 2020; received in revised form December 7, 2020; accepted December 7, 2020.

Correspondence: Petar Risteski, MD, FETCS, Department of Thoracic and Cardiovascular Surgery fohann Wolfgang Goethe University Hospital, Theodor Stern Kai 7, Frankfurt am Main 60590, Germany; +4969-6301-5850; fax: +4969-6301-5849 (e-mail: petarristeski@me.com). of both mitral valve leaflets, as well as two regurgitation jets: a central, wide, high-turbulent jet (Figure 1A) and a second small eccentric jet (Figure 1B) at the level of the anterolateral mitral annulus.

Through an upper mini sternotomy, cardiopulmonary bypass was achieved after aortic and bicaval cannulation. After transverse aortotomy, the bicuspid aortic valve was inspected and revealed a Sievers L-R Type I bicuspid configuration without root dystrophy. Both cusps were not degenerated, without calcification, but prolapsing (the nonseparated cusp more severely than the noncoronary cusp) and the aortic annulus was lightly dilated, as measured with a $26 \mathrm{~mm}$ Hegar dilator. The repair of the bicuspid valve was commenced as follows: the Nodulus Arantius (NA) of the noncoronary cusp was found widened at $8 \mathrm{~mm}$ and recognized as an indicator for cusp prolapse. Using multiple interrupted 6-0 polypropylene sutures, the free margin of the noncoronary cusp was plicated and the width of the NA was reduced to $2 \mathrm{~mm}$, producing an accurate cusp height and subsequently a satisfactory reference point for further adjustment of the nonseparated cusp. Using multiple interrupted 6-0 polypropylene sutures, the nonseparated cusp was plicated centrally to match the height of the reference cusp. A coaptation height from 2 $\mathrm{mm}$ was achieved using the aforementioned techniques. To
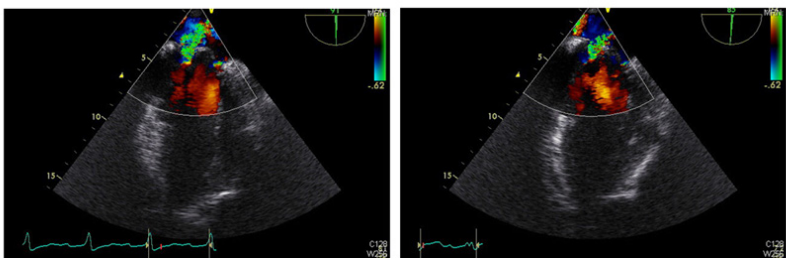

Figure 1. A) Two chamber view showing a major central regurgitation jet and billowing of both leaflets of the mitral valve. B) An additional minor jet is seen originating in close proximity to the mitral annulus.
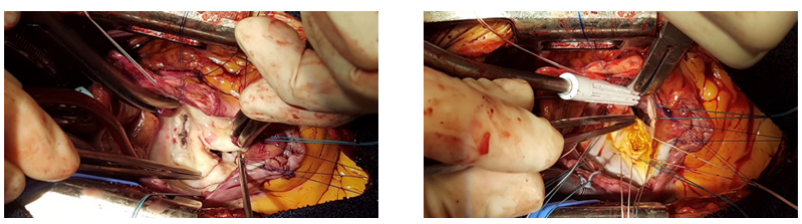

Figure 2. A) Intraoperative photo of a double orifice mitral valve showing a rudimentary additional opening in vicinity of the anterior commissure and the $\mathrm{P} 1$ segment of the mitral valve. B) Close to the base of the $\mathrm{P} 2$ segment, a $1 \times 1 \mathrm{~cm}$ encapsuled annular abscess raised the suspicion of a latent preclinical form of mitral valve endocarditis. 
increase the coaptation height, a continuous annular polytetrafluoroethylene $(\mathrm{CV}-0)$ suture was used to mildly downsize the enlarged aortic annulus to a $23 \mathrm{~mm}$ Hegar dilator and stabilize the basal ring, as recommended [Schneider 2017]. Finally, a coaptation height of $4 \mathrm{~mm}$ was achieved, concluding the repair of the aortic valve.

Access to the mitral valve was gained through an extended transseptal biatriotomy. A detailed examination revealed a highly complex valve anatomy, including a DOMV with a second small "hole-type" orifice at the level of the anterolateral commissure (Figure 2A). The main orifice was attached to only one well-differentiated posteromedial papillary muscle, thus confirming the known parachute configuration. Systematic inspection at this level revealed a generalized myxoid degeneration of the leaflets with multiple billowing and prolapsing segments (P1, A1, A2 and the anterior commissure), chordal elongation and rupture in addition to moderate annular dilatation.

The smaller mitral valve orifice had rudimentary cusps and the chordae were redundantly inserted on the muscular trabeculae of the free ventricular wall. At the base of the P2 segment, a $1 \mathrm{x} 1 \mathrm{~cm}$ encapsuled annular abscess raised the suspicion of a latent preclinical form of mitral valve endocarditis (Figure 2B).

The abscess was incised, drained, and obliterated. The rudimentary "hole-type" second mitral valve orifice was directly sutured, and the orifice was obliterated. The repair of the parachute mitral valve was performed using the P3 segment as the reference point. A total of 8 neochordae (4-0 polytetrafluoroethylene) sutures were used to correct the complex prolapse pathology. The mitral valve reconstruction was completed using a $36 \mathrm{~mm}$ Cosgrove band. Total cardiopulmonary bypass time was 178 minutes, and aortic crossclamp time was 138 minutes.

Transesophageal echocardiographic control, performed shortly after weaning from cardiopulmonary bypass, revealed competent function of both valves. The intraoperative raised suspicion that mitral valve endocarditis could be confirmed soon after as the samples taken from the para-annular abscess were positive for Staphylococcus epidermidis, thus imposing the continuation and adjusting of the already initiated antibiotic treatment. At one year follow up, echocardiographic control confirmed normal function of both valves.

\section{DISCUSSION}

Shone's complex was first described in 1963 by Shone and associates as four left-sided, usually obstructive, heart defects: coarctation of the aorta, subaortic obstruction, parachute mitral valve, and supravalvular mitral ring [Shone 1963]. It is a rare condition, with a described incidence of $0.67 \%$ of all congenital cardiac diseases. Incomplete forms of Shone's complex consisting of only three of the originally described heart defects, prevail in clinical practice [Aslam 2017; Shih 2019].

The present case reveals a unique series of features that had been scarcely described before. A double orifice mitral valve accompanying an incomplete form of Shone's complex is noteworthy as only very few cases have been described in the literature [Kocaba 2014; Salas 1981]. Barlow's disease is a degenerative process that affects the entire mitral valve apparatus. Due to the paucity of such cases in adult patients, Barlow's disease has been described only once before in a patient with DOMV [Chen 2018].

Double orifice mitral valve is a very rare condition defined as a single fibrous annulus with two orifices. It affects both mitral valves and the subvalvular apparatus and is usually associated with other cardiac disorders. Three classes of DOMV prevail: incomplete bridge type (a small stand of tissue connects the anterior and posterior leaflet edges), complete bridge type (the mitral valve is completely divided in two equal or unequal orifices by a fibrous bridge), and hole type (an additional orifice is included in one of the mitral valve commissures). DOMV takes a variety of clinical forms, mostly depending on the associating cardiac defects. It is, however, uncommon to remain clinically silent until adulthood; most cases are reported in infancy.

Our patient tolerated well the small regurgitation jet, resulting from the likely always redundant hole-type mitral valve orifice. The larger mitral orifice and bicuspid aortic valve were not relevantly dysfunctional throughout his life, up to the point when the process of myxoid degeneration and the superimposed aortic regurgitation secondary to arterial hypertension triggered the aforementioned symptoms.

As technical progress constantly improves standard screening measures, more asymptomatic or oligosymptomatic adult patients with rare cardiac malformations are nowadays diagnosed [Shehatha 2018; Shih 2019]. Increasing patient awareness raises the bar and challenges the cardiac surgeon to expand the boundaries of conventional cardiac surgery. Minimally invasive repair of DOMV and bicuspid aortic valve is achievable with excellent early outcome.

\section{REFERENCES}

Aslam S, Khairy P, Shohoudi A, et al. 2017. Shone complex: an underrecognized congenital heart disease with substantial morbidity in adulthood. Can J Cardiol 33(2):253- 259.

Chen Z, Kong WKF, Tay ELW, Petersson F, Poh KK. 2018. Doubleorifice Barlow's mitral valve. Eur Heart J. 39(14):1208-1209.

Greenfield WS. 1876. Double mitral valve. Transactions of the Pathological Society of London. 27:128-129.

Kocabaş A, Ekici F, Çetin İ, Aktaş D. 2014. Three-dimensional echocardiographic evaluation of a patient with double-orifice mitral valve, bicuspid aortic valve, and coarctation of aorta. Echocardiography. 31(1):E33-4.

Salas J, Prieto JA, De Vega G, et al. 1981. Mitral valve with double orifice associated with a bicuspid aortic valve and coarctation of the aorta. Apropos of a case. Rev Esp Cardiol. 34(1):85-8.

Schneider U, Hofmann C, Aicher D, et al. 2017. Suture annuloplasty significantly improves the durability of bicuspid aortic valve repair. Ann Thorac Surg 103(2):504-510.

Shehatha JS, Taha AY, Mirza AJ. 2018. Late Shone complex: a case report and literature review. Journal of Egyptian Society of Cardio-Thoracic Surgery. 26(2):133-135. 
Shih BC, Lim JH, Min J, Kim ER, Kwak JG, Kim WH. 2019. Incomplete form of Shone complex in adult congenital heart disease patient. Korean J Thorac Cardiovasc Surg 52(2):100-104.
Shone JD, Sellers RD, Anderson RC, et al. 1963. The developmental complex of "parachute mitral valve," supravalvular ring of left atrium, subaortic stenosis, and coarctation of aorta. Am J Cardiol 11:714-725. 